\title{
DYNAMICS OF A HIGHER DIMENSIONAL ANALOG OF THE TRIGONOMETRIC FUNCTIONS
}

\author{
Walter Bergweiler ${ }^{\dagger}$ and Alexandre Eremenko ${ }^{\ddagger}$ \\ Christian-Albrechts-Universität zu Kiel, Mathematisches Seminar \\ Ludewig-Meyn-Str. 4, D-24098 Kiel, Germany; bergweiler@math.uni-kiel.de \\ Purdue University, Department of Mathematics \\ West Lafayette, IN 47907-2067, U.S.A.; eremenko@math.purdue.edu
}

\begin{abstract}
We introduce a quasiregular analog $F$ of the sine and cosine function such that, for a sufficiently large constant $\lambda$, the map $x \mapsto \lambda F(x)$ is locally expanding. We show that the dynamics of this map define a representation of $\mathbf{R}^{d}, d \geq 2$, as a union of simple curves $\gamma:[0, \infty) \rightarrow \mathbf{R}^{d}$ which tend to $\infty$ and whose interiors $\gamma^{*}=\gamma((0, \infty))$ are disjoint such that the union of all $\gamma^{*}$ has Hausdorff dimension 1.
\end{abstract}

\section{Introduction and statement of results}

The Julia set $J(f)$ of an entire function $f$ is defined as the set of all points in $\mathbf{C}$ where the iterates $f^{k}$ of $f$ do not form a normal family. An equivalent definition was given in [9]: $J(f)=\partial I(f)$ where $I(f)=\left\{z: f^{n}(z) \rightarrow \infty\right\}$ is the set of escaping points; see [3] for an introduction to the dynamics of entire and meromorphic functions.

Devaney and Krych [7] showed that $J\left(\lambda e^{z}\right)$ is a "Cantor bouquet" for $0<\lambda<1 / e$. To give a precise statement of their result we say that a subset $H$ of $\mathbf{C}$ (or $\mathbf{R}^{d}$ ) is a hair if there exists a continuous injective map $\gamma:[0, \infty) \rightarrow \mathbf{C}$ (or $\mathbf{R}^{d}$ ) such that $\lim _{t \rightarrow \infty} \gamma(t)=\infty$ and $\gamma([0, \infty))=H$. We call $\gamma(0)$ the endpoint of the hair.

The result of Devaney and Krych is the following.

Theorem A. Let $0<\lambda<1 / e$. Then $J\left(\lambda e^{z}\right)$ is an uncountable union of pairwise disjoint hairs.

We denote by $\operatorname{dim} X$ the Hausdorff dimension of a set $X$ in $\mathbf{C}$ (or in $\mathbf{R}^{d}$ ). The following result is due to McMullen [16, Theorem 1.2].

Theorem B. For $\lambda \in \mathbf{C} \backslash\{0\}$ we have $\operatorname{dim} J\left(\lambda e^{z}\right)=2$.

Karpińska [14, Theorem 1.1] proved the following surprising result.

Theorem C. Let $0<\lambda<1 / e$ and let $E_{\lambda}$ be the set of endpoints of the hairs that form $J\left(\lambda e^{z}\right)$. Then $\operatorname{dim} E_{\lambda}=2$ and $\operatorname{dim}\left(J\left(\lambda e^{z}\right) \backslash E_{\lambda}\right)=1$.

The conclusion of Theorem B holds more generally for entire functions of finite order for which the set of critical and asymptotic values is bounded; see [2, Theorem A] and [23]. If, in addition, this set is compactly contained in the immediate

doi:10.5186/aasfm.2011.3610

2010 Mathematics Subject Classification: Primary 30D05, 30C65, 37F10, 37F35.

Key words: Dynamics of entire functions, quasiregular map, Zorich map, Julia set, escaping set, Devaney hair, Hausdorff dimension.

†Supported by the EU Research Training Network CODY, the ESF Networking Programme HCAA and the Deutsche Forschungsgemeinschaft, Be 1508/7-1.

${ }^{\ddagger}$ Supported by NSF grant DMS-0555279 and by the Humboldt Foundation. 
basin of an attracting fixed point, then the conclusions of Theorems $\mathrm{A}$ and $\mathrm{C}$ also hold [1, 2].

These results apply in particular to trigonometric functions. However, the analogue of Theorem A for trigonometric functions had been obtained already much earlier by Devaney and Tangerman [8].

Theorem D. Let $0<\lambda<1$. Then $J(\lambda \sin z)$ is an uncountable union of pairwise disjoint hairs.

McMullen [16, Theorem 1.1] and Karpińska [13, Theorem 3] also considered the case of trigonometric functions. Their results are as follows. Here area $X$ stands for the Lebesgue measure of a measurable subset $X$ of $\mathbf{C}$.

Theorem E. Let $\lambda, \mu \in \mathbf{C}, \lambda \neq 0$. Then area $J(\lambda \sin z+\mu)>0$.

Theorem F. For $0<\lambda<1$ let $E_{\lambda}$ be the set of endpoints of hairs that form $J(\lambda \sin z)$. Then area $E_{\lambda}>0$.

The argument in [14] shows that under the hypothesis of Theorem $\mathrm{F}$ we also have $\operatorname{dim}\left(J(\lambda \sin z) \backslash E_{\lambda}\right)=1$.

The conclusions of Theorems $D$ and $F$, as well as the last remark, hold more generally for functions of the form $f(z)=\lambda \sin z+\mu$ if the parameters are chosen such that the critical values $\pm \lambda+\mu$ of $f$ are contained in the immediate basin of the same attracting fixed point. If this condition on the critical values is not satisfied, then the hairs in the Julia set of $f$ still may exist, but in general distinct hairs may share their endpoints [20].

If the critical values of $f(z)=\lambda \sin z+\mu$ are strictly preperiodic, then $J(f)=\mathbf{C}$. Schleicher ([21], see also [22]) showed that $J(f)$ is still a union of hairs which are pairwise disjoint except for their endpoints, and the Hausdorff dimension of the hairs without their endpoints is 1 . Thus he obtained the following result.

Theorem G. There exists a representation of the complex plane $\mathbf{C}$ as a union of hairs with the following properties:

- the intersection of two hairs is either empty or consists of the common endpoint;

- the union of the hairs without their endpoints has Hausdorff dimension 1.

Zorich [25] introduced a quasiregular analog $F: \mathbf{R}^{3} \rightarrow \mathbf{R}^{3} \backslash\{0\}$ of the exponential function. It was shown in [4] that the results about the dynamics of the exponential function quoted above (Theorems A, B and C) have analogs in the context of Zorich maps.

In this paper we introduce a higher dimensional analog of the trigonometric functions. The dynamics of this map are then used to extend Theorem G to all dimensions greater than 1 .

Theorem 1. For each $d \in \mathbf{N}, d \geq 2$, there exists a representation of $\mathbf{R}^{d}$ as a union of hairs with the following properties:

- the intersection of two hairs is either empty or consists of the common endpoint;

- the union of the hairs without their endpoints has Hausdorff dimension 1.

The construction of our higher dimensional analog of the trigonometric functions is similar to the construction of Zorich's map as given in [12, Section 6.5.4]. We begin 
with a bi-Lipschitz map $F$ from the half-cube

$$
\left\{x=\left(x_{1}, \ldots, x_{d}\right) \in \mathbf{R}^{d}:\|x\|_{\infty} \leq 1, x_{d} \geq 0\right\}=[-1,1]^{d-1} \times[0,1]
$$

to the upper half-ball

$$
\left\{x \in \mathbf{R}^{d}:\|x\|_{2} \leq 1, x_{d} \geq 0\right\}
$$

which maps the face $[-1,1]^{d-1} \times\{1\}$ to the hemisphere $\left\{x \in \mathbf{R}^{d}:\|x\|_{2}=1, x_{d} \geq 0\right\}$. We will give an explicit construction of such a bi-Lipschitz map $F$ in Section 4 . Next we define $F:[-1,1]^{d-1} \times(1, \infty) \rightarrow \mathbf{R}^{d}$ by

$$
F(x)=\exp \left(x_{d}-1\right) F\left(x_{1}, \ldots, x_{d-1}, 1\right) .
$$

The map $F$ is now defined on $[-1,1]^{d-1} \times[0, \infty)$, and it maps $[-1,1]^{d-1} \times[0, \infty)$ bijectively onto the upper half-space $H^{+}:=\left\{x \in \mathbf{R}^{d}: x_{d} \geq 0\right\}$. Using repeated reflections at hyperplanes we can extend $F$ to a map $F: \mathbf{R}^{d} \rightarrow \mathbf{R}^{d}$.

It turns out that the map $F$ is quasiregular. However, we shall not actually use this fact. On the other hand, the quasiregularity of $F$ is one of the underlying ideas in the proofs, and thus we make some remarks about quasiregular maps in Section 5. We also show there that our map $F$ is indeed quasiregular.

We note that since $F$ is locally bi-Lipschitz, the restriction of $F$ to any line is absolutely continuous, and $F$ is differentiable almost everywhere. We denote by

$$
\|D F(x)\|:=\sup _{\|y\|=1}\|D F(x)(y)\|
$$

the operator norm of the derivative $D F(x)$. (Here and in the following $\|y\|=\|y\|_{2}$ for $y \in \mathbf{R}^{d}$; that is, unless specified otherwise we consider the Euclidean norm in $\mathbf{R}^{d}$.) We also put

$$
\ell(D F(x)):=\inf _{\|y\|=1}\|D F(x)(y)\| .
$$

We note that it follows from the definition of $F$ that if $x, x^{\prime} \in(-1,1)^{d-1} \times(1, \infty)$ and $x_{j}=x_{j}^{\prime}$ for $1 \leq j \leq d-1$, then

$$
D F\left(x^{\prime}\right)=\exp \left(x_{d}^{\prime}-x_{d}\right) D F(x)
$$

whenever these derivatives exist.

It is easy to see that

$$
\beta:=\underset{x \in \mathbf{R}^{d}}{\operatorname{essinf}} \ell(D F(x))>0
$$

for our map $F$. We choose $\lambda>1 / \beta$ and consider the map $f=\lambda F$. Clearly $f$ is quasiregular and

$$
\alpha:=\underset{x \in \mathbf{R}^{d}}{\operatorname{ess} \inf } \ell(D f(x))=\lambda \beta>1,
$$

that is, $f$ is locally uniformly expanding in $\mathbf{R}^{d}$.

We put $S:=\mathbf{Z}^{d-1} \times\{-1,1\}$ and for $r=\left(r_{1}, \ldots, r_{d}\right) \in S$ we define

$$
T(r):=\left\{x \in \mathbf{R}^{d}:\left|x_{j}-2 r_{j}\right| \leq 1 \text { for } 1 \leq j \leq d-1, r_{d} x_{d} \geq 0\right\} .
$$

We find that if

$$
\sigma(r):=\sum_{j=1}^{d-1} r_{j}+\frac{1}{2}\left(r_{d}-1\right)
$$

is even, then $f$ maps $T(r)$ bijectively onto $H^{+}$. If $\sigma(r)$ is odd, then $f$ maps $T(r)$ bijectively onto $H^{-}:=\left\{x \in \mathbf{R}^{d}: x_{d} \leq 0\right\}$. 
For a sequence $\underline{s}=\left(s_{k}\right)_{k \geq 0}$ of elements of $S$ we put

$$
H(\underline{s}):=\left\{x \in \mathbf{R}^{d}: f^{k}(x) \in T\left(s_{k}\right) \text { for all } k \geq 0\right\} .
$$

Evidently $\mathbf{R}^{d}=\sum_{\underline{s} \in \underline{S}} H(\underline{s})$, where $\underline{S}$ is the set of all sequences with elements in $S$ for which $H(\underline{s})$ is not empty.

Proposition 1. If $\underline{s} \in \underline{S}$, then $H(\underline{s})$ is a hair.

For $\underline{s} \in \underline{S}$ we denote by $E(\underline{s})$ the endpoint of $H(\underline{s})$.

Proposition 2. If $\underline{s}^{\prime} \neq \underline{s}^{\prime \prime}$, then $H\left(\underline{s}^{\prime}\right) \cap H\left(\underline{s}^{\prime \prime}\right)=\emptyset$ or $H\left(\underline{s}^{\prime}\right) \cap H\left(\underline{s}^{\prime \prime}\right)=\left\{E\left(\underline{s}^{\prime}\right)\right\}=$ $\left\{E\left(\underline{s}^{\prime \prime}\right)\right\}$.

Proposition 3. $\operatorname{dim}\left(\bigcup_{\underline{s} \in \underline{S}} H(\underline{s}) \backslash\{E(\underline{s})\}\right)=1$.

Theorem 1 follows from these propositions.

Acknowledgment. We thank the referee for valuable comments.

\section{Preliminaries}

It follows from the definition of $F$ that

$$
\|F(x)\|=\exp \left(\left|x_{d}\right|-1\right), \quad x \in \mathbf{R}^{d},\left|x_{d}\right| \geq 1,
$$

so that

$$
\|f(x)\|=\lambda \exp \left(\left|x_{d}\right|-1\right), \quad x \in \mathbf{R}^{d},\left|x_{d}\right| \geq 1 .
$$

For $r \in S$ we denote by $\Lambda^{r}$ the inverse function of $\left.f\right|_{T(r)}$. Thus $\Lambda^{r}: H^{+} \rightarrow T(r)$ or $\Lambda^{r}: H^{-} \rightarrow T(r)$, depending on whether $\sigma(r)$ is even or odd. For $x \in T(r)$ and $y=f(x)$ we have

and thus

$$
\left\|D \Lambda^{r}(y)\right\|=\frac{1}{\ell(D f(x))}
$$

$$
\left\|D \Lambda^{r}(y)\right\| \leq \frac{1}{\alpha}
$$

by (1.2). It follows from (2.2) that if $a, b \in T(r)$, then

$$
\|a-b\|=\left\|\Lambda^{r}(f(a))-\Lambda^{r}(f(b))\right\| \leq \frac{1}{\alpha}\|f(a)-f(b)\| .
$$

Hence

$$
\|f(a)-f(b)\| \geq \alpha\|a-b\| \quad \text { for } a, b \in T(r), r \in S .
$$

If $\left|x_{d}\right| \geq 1$, then we have

$$
\ell(D f(x)) \geq \alpha \exp \left(\left|x_{d}\right|-1\right)=\frac{\alpha\|f(x)\|}{\lambda}=\beta\|f(x)\|
$$

by (1.1), (1.2) and (2.1). Note that the condition $\left|x_{d}\right| \geq 1$ is equivalent to $\|y\| \geq \lambda$. Thus

$$
\left\|D \Lambda^{r}(y)\right\| \leq \frac{1}{\beta\|y\|}, \quad y \in \mathbf{R}^{d},\|y\| \geq \lambda
$$

Similarly we deduce from (1.1) that there exists a positive constant $\delta$ such that

$$
\ell\left(D \Lambda^{r}(y)(x)\right) \geq \frac{\delta}{\|y\|}, \quad y \in \mathbf{R}^{d},\|y\| \geq \lambda .
$$


We shall also need the following result.

Lemma 1. Let $\underline{s}=\left(s_{k}\right)_{k \geq 0}$ be an element of $\underline{S}$ and let $x, y \in H(\underline{s})$. For $k \geq 0$ we put $x^{k}=\left(x_{1}^{k}, \ldots, x_{d}^{k}\right):=f^{k}(x)$ and $y^{k}=\left(y_{1}^{k}, \ldots, y_{d}^{k}\right):=f^{k}(y)$.

There exists $M>0$ with the following property: if

$$
\left|y_{d}^{k}\right|>\left|x_{d}^{k}\right|+M
$$

for some $k \geq 0$, then

$$
\left|y_{d}^{k+1}\right|>\frac{\lambda}{3} \exp \left|y_{d}^{k}\right|+M \geq 5\left|x_{d}^{k+1}\right|+M .
$$

Proof. We will denote by $p$ the projection

$$
p: \mathbf{R}^{d} \rightarrow \mathbf{R}^{d-1},\left(x_{1}, \ldots, x_{d-1}, x_{d}\right) \mapsto\left(x_{1}, \ldots, x_{d-1}\right) .
$$

Since $\left|x_{j}^{k}-y_{j}^{k}\right| \leq 2$ for $1 \leq j \leq d-1$ and all $k$ we have

$$
\left\|p\left(x^{k}\right)-p\left(y^{k}\right)\right\| \leq 2 \sqrt{d-1}
$$

for all $k$.

Suppose now that (2.6) holds. Then using (2.1) and (2.7) we obtain

$$
\begin{aligned}
\left|y_{d}^{k+1}\right| & \geq\left\|y^{k+1}\right\|-\left\|p\left(y^{k+1}\right)\right\| \\
& \geq \lambda \exp \left(\left|y_{d}^{k}\right|-1\right)-\left\|p\left(x^{k+1}\right)\right\|-2 \sqrt{d-1} \\
& \geq \lambda \exp \left(\left|y_{d}^{k}\right|-1\right)-\lambda \exp \left(\left|x_{d}^{k}\right|-1\right)-2 \sqrt{d-1} \\
& \geq \lambda \exp \left(\left|y_{d}^{k}\right|-1\right)-\lambda \exp \left(\left|y_{d}^{k}\right|-M-1\right)-2 \sqrt{d-1} \\
& =\frac{\lambda}{e}\left(1-e^{-M}\right) \exp \left|y_{d}^{k}\right|-2 \sqrt{d-1} .
\end{aligned}
$$

Noting that $\left|y_{d}^{k}\right|>M$ by (2.6) we find that if $M$ is sufficiently large, then

$$
\left|y_{d}^{k+1}\right| \geq \frac{\lambda}{3} \exp \left|y_{d}^{k}\right|+M
$$

Since

$$
\frac{\lambda}{3} \exp \left|y_{d}^{k}\right|>\frac{\lambda}{3} e^{M} \exp \left|x_{d}^{k}\right|=\frac{e}{3} e^{M}\left\|x^{k+1}\right\| \geq \frac{e}{3} e^{M}\left|x_{d}^{k+1}\right|,
$$

the last inequality in the conclusion of the lemma also holds if $M$ is large.

\section{Proof of the Propositions}

Proof of Proposition 1. For a sequence $\underline{s}=\left(s_{k}\right)$ in $\underline{S}$ we have

$$
H(\underline{s})=\bigcap_{k \geq 0}\left(\Lambda^{s_{0}} \circ \Lambda^{s_{1}} \circ \ldots \circ \Lambda^{s_{k}}\right)\left(T\left(s_{k+1}\right)\right) .
$$

Thus $X:=H(\underline{s}) \cup\{\infty\}$ is an intersection of nested, connected, compact subsets of $\overline{\mathbf{R}^{d}}:=\mathbf{R}^{d} \cup\{\infty\}$. This implies that $X$ is compact and connected.

To prove that $H(\underline{s})$ is a hair we follow Rottenfußer, Rückert, Rempe and Schleicher [19] and use the following lemma from [17].

Lemma 2. Let $X$ be a non-empty, compact, connected metric space. Suppose that there is a strict linear ordering $\prec$ on $X$ such that the order topology on $X$ agrees with the metric topology. Then either $X$ consists of a single point or there is an order-preserving homeomorphism from $X$ onto $[0,1]$. 
To define the linear ordering on $X=H(\underline{s}) \cup\{\infty\}$ we choose $M$ according to Lemma 1. For $x, y \in H(\underline{s})$ we say that $x \prec y$ if there exists $k \geq 0$ such that $\left|y_{d}^{k}\right|>\left|x_{d}^{k}\right|+M$, and we define $x \prec \infty$ for all $x \in H(\underline{s})$. Lemma 1 implies that $x \prec y$ and $y \prec x$ cannot hold simultaneously. Another easy consequence of Lemma 1 is that our relation $\prec$ is transitive.

To show that it is a linear ordering we notice that $\left\|x^{k}-y^{k}\right\| \geq \alpha^{k}\|x-y\|$ by (2.3). Using (2.7) we obtain

$$
\left|x_{d}^{k}-y_{d}^{k}\right| \geq\left\|x^{k}-y^{k}\right\|-\left\|p\left(x^{k}\right)-p\left(y^{k}\right)\right\| \geq \alpha^{k}\|x-y\|-2 \sqrt{d-1} .
$$

Thus $x \neq y$ implies either $x \prec y$ or $y \prec x$.

Now we prove that the order topology on $X$ is the same as the topology induced from $\overline{\mathbf{R}^{d}}$. We have to show that the identity map from $X$ with the induced topology to $X$ with the order topology is a homeomorphism. Since $X$ with the induced topology is compact and since $X$ with the order topology is Hausdorff, it suffices to show that the identity map is continuous [15, p. 141, Theorem 8]. Thus we only have to show that the sets

$$
U^{-}(a):=\{w \in X: w \prec a\} \quad \text { and } \quad U^{+}(a):=\{w \in X: w \succ a\}
$$

are open with respect to the induced topology for all $a \in X$. In order to do so, let $w \in U^{-}(a)$ and choose the minimal $k$ such that $\left|w_{d}^{k}\right|<\left|a_{d}^{k}\right|-M$. Then there is a neighborhood $V$ of $w$ in $\mathbf{R}^{d}$ where the same inequality is satisfied. The intersection $V \cap H(\underline{s})$ is a neighborhood of $w$ that is contained in $U^{-}(a)$. Thus $U^{-}(a)$ is open with respect to the induced topology. The proof for $U^{+}(a)$ is similar.

Thus the order topology on $X$ agrees with the topology induced from $\overline{\mathbf{R}^{d}}$. Proposition 1 now follows from Lemma 2.

Proof of Proposition 2. Let $y \in H\left(\underline{s}^{\prime}\right) \cap H\left(\underline{s}^{\prime \prime}\right)$. Let $m$ be the smallest subscript such that $s_{m}^{\prime} \neq s_{m}^{\prime \prime}$. Then $f^{m}(y)$ belongs to the common boundary of $T\left(s_{m}^{\prime}\right)$ and $T\left(s_{m}^{\prime \prime}\right)$. From the definition of $f$ we conclude that $f^{k}(y)$ belongs to the hyperplane $\left\{x \in \mathbf{R}^{d}: x_{d}=0\right\}$ for all $k \geq m+1$. This implies that $x \prec y$ is impossible for any $x$. So $y$ is the minimal element of the order $\prec$ and thus an endpoint of $H\left(\underline{s}^{\prime}\right)$ and $H\left(\underline{s}^{\prime \prime}\right)$.

Proof of Proposition 3. We follow the argument in [4] and with $\psi:[1, \infty) \rightarrow \mathbf{R}$,

$$
\psi(t):=\exp (\sqrt{\log t})
$$

and $L:=\max \{e, 4 \lambda\}$ we put

We then have

$$
\Omega:=\left\{x \in \mathbf{R}^{d}:\left|x_{d}\right| \geq L,\|p(x)\| \leq \psi\left(\left|x_{d}\right|\right)\right\} .
$$

$$
\|x\| \leq\left|x_{d}\right|+\|p(x)\| \leq\left|x_{d}\right|+\psi\left(\left|x_{d}\right|\right) \leq 2\left|x_{d}\right|, \quad x \in \Omega .
$$

The following result is analogous to [4, Lemma 5.3].

Lemma 3. If $y \in H(\underline{s}) \backslash\{E(\underline{s})\}$ then $f^{k}(y) \rightarrow \infty$ as $k \rightarrow \infty$. Moreover, we have $f^{k}(y) \in \Omega$ for all large $k$.

Proof. Let $\underline{s}=\left(s_{k}\right)_{k \geq 0} \in \underline{S}$ such that $y \in H(\underline{s})$. With $x=E(\underline{s})$ and the ordering $\prec$ as in Section 3 we have $x \prec y$. As before, we put $x^{k}=f^{k}(x)$ and $y^{k}=f^{k}(y)$ for $k \geq 0$. By Lemma 1 we have

$$
\left|y_{d}^{k}\right| \geq 5\left|x_{d}^{k}\right|+M
$$


for all large $k$. Using (3.1) we see that $\left|y_{d}^{k}\right| \rightarrow \infty$ and hence $y^{k} \rightarrow \infty$ as $k \rightarrow \infty$.

Since

$$
\left\|p\left(y^{k}\right)\right\| \leq\left\|p\left(x^{k}\right)\right\|+2 \sqrt{d-1} \leq\left\|x^{k}\right\|+2 \sqrt{d-1}
$$

by (2.7) we see that $f^{k}(y) \in \Omega$ holds for large $k$ if $\left\|x^{k}\right\| \leq R$, where $R$ is any fixed constant. Noting that

$$
\left\|x^{k}\right\| \leq \lambda \exp \left|x_{d}^{k-1}\right| \leq \lambda \exp \left\|x^{k-1}\right\|,
$$

we also find that $f^{k}(y) \in \Omega$ holds for all large $k$ for which $\left\|x^{k-1}\right\| \leq \log (R / \lambda)$.

We may thus suppose that $\min \left\{\left\|x^{k}\right\|,\left\|x^{k-1}\right\|\right\}$ is large. Lemma 1 now yields for large $k$ that

$$
\begin{aligned}
\left|y_{d}^{k-1}\right| & \geq \frac{\lambda}{3} \exp \left|y_{d}^{k-2}\right|+M \geq \frac{\lambda}{3} \exp \left(5\left|x_{d}^{k-2}\right|+M\right)+M \\
& \geq \frac{e^{M}}{3 \lambda^{4}}\left(\lambda \exp \left|x_{d}^{k-2}\right|\right)^{5}+M \geq\left\|x^{k-1}\right\|^{4} \geq\left|x_{d}^{k-1}\right|^{4},
\end{aligned}
$$

and hence that

$$
\begin{aligned}
\left|y_{d}^{k}\right| & \geq \frac{\lambda}{3} \exp \left|y_{d}^{k-1}\right|+M \geq \frac{\lambda}{3} \exp \left(\left|x_{d}^{k-1}\right|^{4}\right) \\
& \geq \frac{\lambda}{3} \exp \left(\left(\log \left\|x^{k}\right\|\right)^{4}\right) \geq \exp \left(\left(\log \left\|x^{k}\right\|\right)^{3}\right) .
\end{aligned}
$$

Thus

$$
\begin{aligned}
\left\|p\left(y^{k}\right)\right\| & \leq\left\|p\left(x^{k}\right)\right\|+2 \sqrt{d-1} \leq\left\|x^{k}\right\|+2 \sqrt{d-1} \\
& \leq \exp \left(\left(\log \left|y_{d}^{k}\right|\right)^{1 / 3}\right)+2 \sqrt{d-1} \leq \exp \sqrt{\log \left|y_{d}^{k}\right|}
\end{aligned}
$$

for large $k$. This means that $y_{k} \in \Omega$, and the proof of Lemma 3 is completed.

The following result [4, Lemma 5.2] is a simple consequence of some classical covering lemmas. Here we denote by $B(x, r)$ the open ball of radius $r$ around a point $x \in \mathbf{R}^{d}$.

Lemma 4. Let $Y \subset \mathbf{R}^{d}$ and $\rho>1$. Suppose that for all $y \in Y$ and $\eta>0$ there exist $r(y) \in(0,1), d(y) \in(0, \eta)$ and $N(y) \in \mathbf{N}$ satisfying $d(y)^{\rho} N(y) \leq r(y)^{d}$ such that $B(y, r(y)) \cap Y$ can be covered by $N(y)$ sets of diameter at most $d(y)$. Then $\operatorname{dim} Y \leq \rho$.

In [4, Lemma 5.2] it is additionally assumed that $Y$ is bounded, but this hypothesis can be omitted, since the Hausdorff dimension of a set is the supremum of the Hausdorff dimensions of its bounded subsets.

We now begin with the actual proof of Proposition 3, following the argument in [4]. Since $f$ is locally bi-Lipschitz, and since the Hausdorff dimension is invariant under bi-Lipschitz maps, Lemma 3 implies that it suffices to show that

$$
Y:=\left\{y \in H(\underline{s}) \backslash\{E(\underline{s})\}: f^{k}(y) \in \Omega \text { for all } k \geq 0\right\}
$$

has Hausdorff dimension 1. We shall prove this using Lemma 4.

Let $y \in Y \cap H(\underline{s})$ and, as before, put $y^{k}=f^{k}(y)$. With $x=E(\underline{s})$ we deduce from Lemma 1 that

$$
\left|y_{d}^{j+1}\right|>\frac{\lambda}{3} \exp \left|y_{d}^{j}\right|+M
$$

for large $j$. 
We now fix a large $k$ and denote by $B_{k}$ the closed ball of radius $\frac{1}{2}\left|y_{d}^{k}\right|$ around $y_{k}$. We cover $B_{k} \cap \Omega$ by closed cubes of sidelength 1 lying in $\left\{x \in \mathbf{R}^{d}:\left|x_{d}\right| \geq \frac{1}{2}\left|y_{d}^{k}\right|\right\}$. If $c>2^{d-1}$, then the number $N_{k}$ of cubes required satisfies

$$
N_{k} \leq c\left|y_{d}^{k}\right| \psi\left(2\left|y_{d}^{k}\right|\right)^{d-1}
$$

provided $k$ is large enough. Given $\varepsilon>0$ we thus can achieve that

$$
N_{k} \leq\left|y_{d}^{k}\right|^{1+\varepsilon}
$$

by choosing $k$ large.

Let $B_{0}$ be the component of $f^{-k}\left(B_{k}\right)$ that contains $y$. With

$$
\varphi:=\Lambda^{s_{0}} \circ \Lambda^{s_{1}} \circ \cdots \circ \Lambda^{s_{k-1}}
$$

we have $B_{0}=\varphi\left(B_{k}\right)$. Using (2.2) and (2.4) we find that if $C$ is one of the cubes of sidelength 1 used to cover $B_{k} \cap \Omega$, then

$$
\operatorname{diam} \varphi(C) \leq \frac{1}{\alpha^{k-1}} \frac{2}{\beta\left|y_{d}^{k}\right|} \operatorname{diam} C \leq \frac{1}{\left|y_{d}^{k}\right|}
$$

if $k$ is sufficiently large. Thus we can cover $B_{0} \cap Y$ by $N_{k}$ sets of diameter $d_{k}$, where

$$
d_{k} \leq \frac{1}{\left|y_{d}^{k}\right|}
$$

In order to apply Lemma 4 we estimate the radius $r_{k}$ of the largest ball around $y$ that is contained in $B_{0}$. Let $z \in \partial B_{0}$ with $\|z-y\|=r_{k}$ and let $\sigma_{0}$ be the straight line connecting $y$ and $z$. For $1 \leq j \leq k$ we put $\sigma_{j}=f^{j}\left(\sigma_{0}\right), B_{j}=f^{j}\left(B_{0}\right)$ and $z^{j}=f^{j}(z)$. Then $\sigma_{k}$ connects $y^{k}$ to $z^{k} \in \partial B_{k}$ and thus

$$
\text { length }\left(\sigma_{k}\right) \geq \frac{1}{2}\left|y_{d}^{k}\right|
$$

We deduce from (2.4) that

$$
\operatorname{diam} B_{k-1}=\operatorname{diam} \Lambda^{s_{k-1}}\left(B_{k}\right) \leq \frac{2}{\beta\left|y_{d}^{k}\right|} \operatorname{diam} B_{k}=\frac{2}{\beta}
$$

and hence

$$
\operatorname{diam} B_{j} \leq \frac{2}{\beta}
$$

for $j \leq k-1$ by (2.2). Since $\left|y_{d}^{j}\right| \geq L>4 / \beta$, this implies that

$$
\sigma_{j} \subset B_{j} \subset B\left(y^{j}, \frac{1}{2}\left|y_{d}^{j}\right|\right) \subset B\left(y^{j}, \frac{1}{2}\left\|y^{j}\right\|\right)
$$

for $j \leq k-1$. It thus follows from (2.5) and (3.2) that

$$
\text { length } \sigma_{j}=\operatorname{length} \Lambda^{s_{j}}\left(\sigma_{j+1}\right) \geq \frac{2 \delta}{3\left\|y^{j+1}\right\|} \text { length } \sigma_{j+1} \geq \frac{\delta}{3\left|y_{d}^{j+1}\right|} \text { length } \sigma_{j+1}
$$

for $j \leq k-1$ and this implies that

$$
\text { length } \sigma_{k} \leq\left(\frac{3}{\delta}\right)^{k}\left(\prod_{j=1}^{k}\left|y_{d}^{j}\right|\right) \text { length } \sigma_{0}
$$


Combining this with (3.6) we find that

$$
r_{k}=\text { length } \sigma_{0} \geq \frac{1}{2}\left(\frac{\delta}{3}\right)^{k} \frac{1}{\prod_{j=1}^{k-1}\left|y_{d}^{j}\right|} .
$$

Using (3.3) we see that we can achieve

$$
r_{k} \geq \frac{1}{\left|y_{d}^{k}\right|^{\varepsilon}}
$$

by choosing $k$ large.

We thus find that we can cover $B\left(y, r_{k}\right) \cap Y$ by $N_{k}$ sets of diameter at most $d_{k}$, where $N_{k}, d_{k}$ and $r_{k}$ satisfy (3.4), (3.5) and (3.7). With $\rho=1+(d+1) \varepsilon$ it follows from (3.4), (3.5) and (3.7) that

$$
\left(d_{k}\right)^{\rho} N_{k} \leq\left|y_{d}^{k}\right|^{1+\varepsilon-\rho}=\left|y_{d}^{k}\right|^{-d \varepsilon} \leq\left(r_{k}\right)^{d} .
$$

Given $\eta>0$ we can also achieve that $r_{k}<1$ and $d_{k}<\eta$ by choosing $k$ large. We thus see that the hypothesis of Lemma 4 are satisfied with $r(y)=r_{k}, d(y)=d_{k}$ and $N(y)=N_{k}$.

It follows that $\operatorname{dim} Y \leq \rho=1+(d+1) \varepsilon$. Since $\varepsilon>0$ was arbitrary, we obtain $\operatorname{dim} Y \leq 1$.

\section{An explicit bi-Lipschitz map}

$$
\begin{aligned}
& \text { Let } B^{+}:=[-1,1]^{d-1} \times[0,1], B^{-}:=[-1,1]^{d-1} \times[-1,0], \\
& U^{+}:=\left\{x \in \mathbf{R}^{d}:\|x\|_{2} \leq 1, x_{d} \geq 0\right\} \quad \text { and } \quad U^{-}:=\left\{x \in \mathbf{R}^{d}:\|x\|_{2} \leq 1, x_{d} \leq 0\right\} .
\end{aligned}
$$

Then $h_{1}:=B^{+} \rightarrow B^{-}, x \mapsto x-(0, \ldots, 0,1)$, and $h_{2}: B^{-} \rightarrow U^{-}, x \mapsto\left(\|x\|_{\infty} /\|x\|_{2}\right) x$, are both bi-Lipschitz, and with $X:=[-1,1]^{d-1} \times\{1\}$ and $Y:=\left\{x \in \mathbf{R}^{d}:\|x\|_{2} \leq\right.$ $\left.1, x_{d}=0\right\}$ we have $h_{2}\left(h_{1}(X)\right)=Y$. It remains to define a bi-Lipschitz map $h_{3}: U^{-} \rightarrow$ $U^{+}$with $h_{3}(Y)=\left\{x \in \mathbf{R}^{d}:\|x\|_{2}=1, x_{d} \geq 0\right\}$. Then $h:=h_{3} \circ h_{2} \circ h_{1}$ has the desired properties.

In order to define $h_{3}$ we note that

$$
T(z)=\frac{z+i}{i z+1}
$$

defines a bi-Lipschitz map from the lower half-disk $\{z \in \mathbf{C}:|z| \leq 1, \operatorname{Im} z \leq 0\}$ to the upper half-disc $\{z \in \mathbf{C}:|z| \leq 1, \operatorname{Im} z \geq 0\}$, with $\{z \in \mathbf{C}:|z| \leq 1, \operatorname{Im} z=0\}$ being mapped onto $\{z \in \mathbf{C}:|z|=1, \operatorname{Im} z \geq 0\}$. With $x=\left(x_{1}, \ldots, x_{d}\right)=\left(p(x), x_{d}\right)$ and $z=\|p(x)\|_{2}+i x_{d}$ it follows that

$$
h_{3}(x)=\left(\frac{p(x)}{\|p(x)\|_{2}} \operatorname{Re} T(z), \operatorname{Im} T(z)\right)
$$

has the desired properties.

\section{Quasiregular maps}

Let $\Omega \subset \mathbf{R}^{d}$ be open. A continuous map $f: \Omega \rightarrow \mathbf{R}^{d}$ is called quasiregular if it belongs to the Sobolev space $W_{d, \text { loc }}^{1}(\Omega)$ and if there exists a constant $K_{O} \geq 1$ such that

$$
\|D F(x)\|^{d} \leq K_{O} J_{F}(x) \text { a.e. }
$$


where $J_{F}=\operatorname{det} D F$ denotes the Jacobian determinant. Equivalently, there exists $K_{I} \geq 0$ such that

$$
J_{F}(x) \leq K_{I} \ell(D F(x))^{d} \quad \text { a.e. }
$$

The smallest constants $K_{O}$ and $K_{I}$ for which the above estimates hold are called the outer and inner dilatation. For a thorough treatment of quasiregular maps we refer to [18].

To see that our map $F$ defined in Section 1 is quasiregular, we note first that (5.1) holds on the half-cube $(-1,1)^{d-1} \times(0,1)$ since $F$ is bi-Lipschitz there. By the same reason, (5.1) holds on the bounded set $(-1,1)^{d-1} \times(1,2)$. Using (1.1) we deduce that $(5.1)$ holds on $(-1,1)^{d-1} \times(1, \infty)$. Thus $(5.1)$ holds on $(-1,1)^{d-1} \times(0, \infty)$ and in the sets obtained from this by reflection. We deduce that $F$ is indeed quasiregular.

We mention that it follows from (5.1) and (5.2) that if $F$ is quasiregular, then

$$
\|D F(x)\| \leq K \ell(D F(x)) \text { a.e. }
$$

where $K=\left(K_{O} K_{I}\right)^{1 / d}$. We could also use (5.3) instead of (5.1) or (5.2) in the definition of quasiregularity. It follows from (2.4) and (2.5) that (5.3) holds for $\left|x_{d}\right| \geq 1$ with $K=1 /(\beta \delta)$. This is one reason why we said in the introduction that the quasiregularity of $F$ is among the underlying ideas of the proof.

We note that for quasiregular maps there is no obvious definition of the Julia set; see, however, $[5,24]$. On the other hand, the escaping set $I(f)$ can be defined. It was shown in [6] that if $f$ is a quasiregular self-map of $\mathbf{R}^{d}$ with an essential singularity at $\infty$, then $I(f) \neq \emptyset$. In fact, $I(f)$ has an unbounded component. Fletcher and Nicks [11] have shown that for quasiregular maps of polynomial type the boundary of the escaping set has properties similar to the Julia set of polynomials.

We mention that for the entire functions $f(z)=\lambda e^{z}$ or $\lambda \sin z+\mu$ considered in Theorems A-G we have $I(f) \subset J(f)$ and thus $J(f)=\overline{I(f)}$; see [10, Theorem 1]. This plays an important role in the proofs of these theorems. For example, McMullen actually proved that the conclusion of Theorems B and E holds with $J(f)$ replaced by $I(f)$. Also, a crucial part in the proofs of Theorems C, F and G is based on the fact that points which are on a hair but which are not endpoints escape to infinity under iteration very fast.

This also played an important role in our proof. In particular, for the map $f$ considered in this paper we have

$$
\bigcup_{\underline{s} \in \underline{S}} H(\underline{s}) \backslash\{E(\underline{s})\} \subset I(f)
$$

by Lemma 3. On the other hand, it is not difficult to see that $\{E(\underline{s}): \underline{s} \in \underline{S}\}$ intersects both $I(f)$ and the complement of $I(f)$.

\section{References}

[1] Barański, K.: Trees and hairs for some hyperbolic entire maps of finite order. - Math. Z. 257, 2007, 33-59.

[2] Barański, K.: Hausdorff dimension of hairs and ends for entire maps of finite order. - Math. Proc. Cambridge Philos. Soc. 145, 2008, 719-737.

[3] Bergweiler, W.: Iteration of meromorphic functions. - Bull. Amer. Math. Soc. (N.S.) 29, 1993, 151-188.

[4] Bergweiler, W.: Karpińska's paradox in dimension 3. - Duke Math. J. 154, 2010, 599-630. 
[5] Bergweiler, W.: Iteration of quasiregular maps. - Comput. Methods Funct. Theory 10, 2010, $455-481$.

[6] Bergweiler, W., A. Fletcher, J. Langley, and J. Meyer: The escaping set of a quasiregular mapping. - Proc. Amer. Math. Soc. 137, 2009, 641-651.

[7] Devaney, R. L., and M. Krych: - Dynamics of $\exp (z)$. - Ergodic Theory Dynam. Systems $4,1984,35-52$.

[8] Devaney, R. L., and F. Tangerman: Dynamics of entire functions near the essential singularity. - Ergodic Theory Dynam. Systems 6, 1986, 489-503.

[9] Eremenko, A.: Iteration of entire functions, "Dynamical Systems and Ergodic Theory". Banach Center Publ. 23, Polish Sci. Publ., Warsaw, 1989, 339-345.

[10] Eremenko, A. E., and M. Yu. Lyubich: Dynamical properties of some classes of entire functions. - Ann. Inst. Fourier 42, 1992, 989-1020.

[11] Fletcher, A., and D. A. Nicks: Quasiregular dynamics on the $n$-sphere. - Ergodic Theory Dynam. Systems 31, 2011, 23-31.

[12] Iwaniec, T., and G. Martin: Geometric function theory and non-linear analysis. - Oxford Math. Monogr., Oxford University Press, New York, 2001.

[13] KarpińsKa, B.: Area and Hausdorff dimension of the set of accessible points of the Julia sets of $\lambda e^{z}$ and $\lambda \sin z$. - Fund. Math. 159, 1999, 269-287.

[14] Karpińska, B.: Hausdorff dimension of the hairs without endpoints for $\lambda \exp (z)$. - C. R. Acad. Sci. Paris Ser. I Math. 328, 1999, 1039-1044.

[15] Kelley, J. L.: General topology. - Van Nostrand, Toronto, New York, London, 1955.

[16] McMullen, C.: Area and Hausdorff dimension of Julia sets of entire functions. - Trans. Amer. Math. Soc. 300, 1987, 329-342.

[17] NADler, S.: Continuum theory. An introduction. - Marcel Dekker, NY, 1992.

[18] Rickman, S.: Quasiregular mappings. - Springer, Berlin, Heidelberg, New York, 1993.

[19] Rottenfusser, G., J. RÜCKert, L. Rempe, and D. Schleicher: Dynamic rays of boundedtype entire functions. - Ann. of Math. (2) (to appear).

[20] Rottenfusser, G., and D. Schleicher: Escaping points of the cosine family, "Transcendental Dynamics and Complex Analysis". - London Math. Soc. Lect. Notes Ser. 348, Cambridge Univ. Press, Cambridge, 2008, 396-424.

[21] Schleicher, D.: The dynamical fine structure of iterated cosine maps and a dimension paradox. - Duke Math. J. 136, 2007, 343-356.

[22] Schleicher, D.: Hausdorff dimension, its properties, and its surprises. - Amer. Math. Monthly 114, 2007, 509-528.

[23] Schubert, H.: Über die Hausdorff-Dimension der Juliamenge von Funktionen endlicher Ordnung. - Dissertation, University of Kiel, 2007.

[24] Sun, D., and L. YANG: Quasirational dynamic system. - Chinese Sci. Bull. 45, 2000, 12771279 .

[25] Zorich, V.: A theorem of M. A. Lavrent'ev on quasiconformal space maps. - Sb. Math. 3, 1967, 389-403; Transl. of Mat. Sb. (N.S.) 116, 1967, 417-433 (in Russian). 